\title{
Erratum to: Vegetation change based on SPOT-VGT data from 1998-2007, northern China
}

\author{
Shulin Liu $\cdot$ Tao Wang $\cdot$ Jian Guo $\cdot$ \\ Jianjun Qu $\cdot$ Peijun An
}

Published online: 22 December 2009

(C) Springer-Verlag 2009

\section{Erratum to: Environ Earth Sci}

\section{DOI 10.1007/s12665-009-0281-4}

The original version of this article unfortunately contained two mistakes. In the legends to Fig. 6 and Table 2 it should read $P<0.05$ instead of $P>0.05$.

The correct versions are given below.

The online version of the original article can be found under doi:10.1007/s12665-009-0281-4.

S. Liu $(\bowtie) \cdot$ T. Wang $\cdot$ J. Guo $\cdot$ J. Qu

Key Laboratory of Desert and Desertification,

Cold and Arid Regions Environmental and Engineering

Research Institute, Chinese Academy of Sciences,

Lanzhou 730000, Gansu, China

e-mail: lius1@1zb.ac.cn

P. An

The Lanzhou Branch of the National Science Library,

Chinese Academy of Sciences,

Lanzhou 730000, Gansu, China 
Fig. 6 Change trend spatial distribution of yearly NDVI maximum from 1998-2007 $(P<0.05)$

Table 2 The slope statistics of the yearly MVC NDVI change trend in the northern China from 1998-2007 $(P<0.05)$

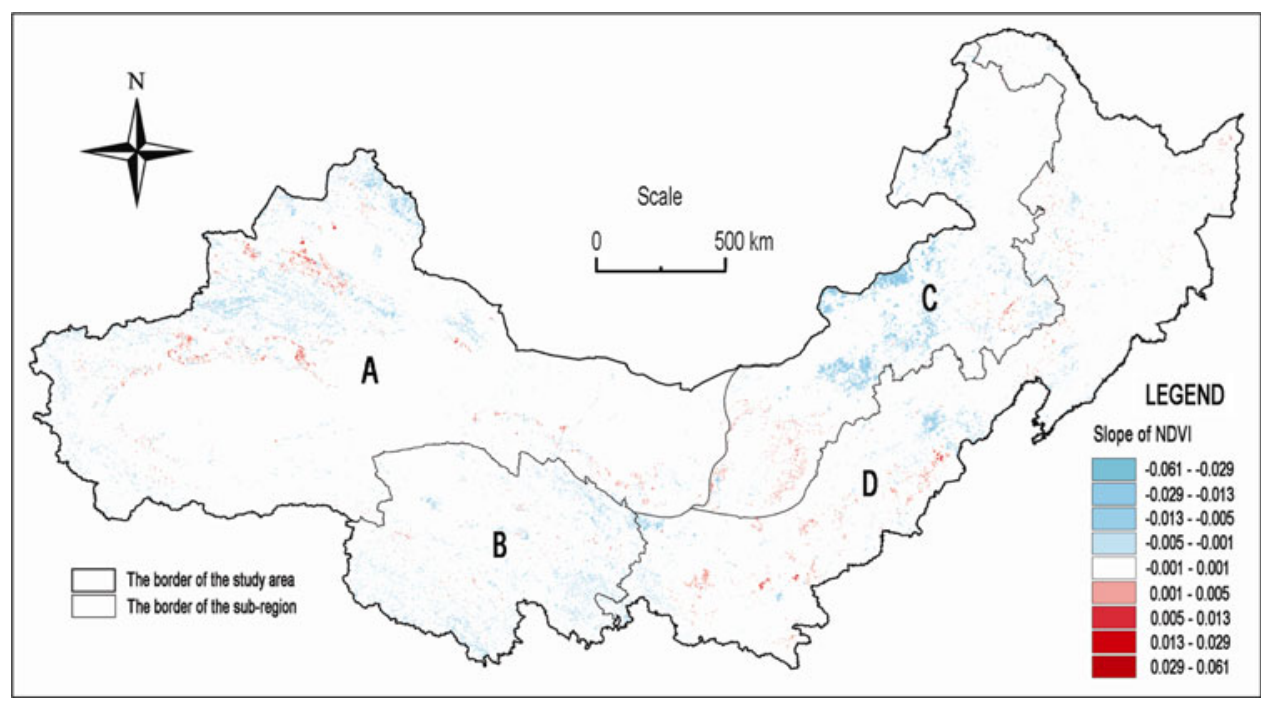

\begin{tabular}{llcc}
\hline Scope of slope value & State & Number of pixels & Area percentage \\
\hline-0.061 to -0.029 & Very serious degradation & 225 & 0.002 \\
-0.029 to -0.013 & Serious degradation & 21,186 & 0.179 \\
-0.013 to -0.005 & Moderate degradation & 96,890 & 0.818 \\
-0.005 to -0.001 & Slight degradation & 106,764 & 0.902 \\
-0.001 to 0.001 & Basically stable & $11,842,359$ & 97.560 \\
0.001 to 0.005 & Slight improvement & 47,428 & 0.400 \\
0.005 to 0.013 & Moderate improvement & 15,938 & 0.135 \\
0.013 to 0.029 & Obvious improvement & 605 & 0.005 \\
0.029 to 0.061 & Very obvious improvement & 0 & 0 \\
\hline
\end{tabular}

